\title{
Editorial
}

\section{Parto programado en el domicilio: mirada desde la SOCHOG}

Bertrand Russell, uno de los principales y más influyentes filósofos del siglo XX, dirigía un mensaje a las futuras generaciones diciendo: "cuando estés estudiando cualquier tema o considerando cualquier filosofía, pregúntate a ti mismo únicamente: ¿cuáles son los hechos? ¿y cuál es la verdad que los hechos sostienen? Nunca te dejes desviar, ya sea por lo que tú deseas creer o por lo que crees que te traería beneficio si así fuese creído. Observa únicamente e indudablemente cuáles son los hechos".

Recomendamos usar este consejo, no solo en la vida en general, sino que también en la medicina, para dirigir nuestro trabajo. La medicina basada en evidencia es el enfoque actual que recomendamos para la práctica médica, optimizando la toma de decisiones, de acuerdo a sólida información científica. La medicina basada en evidencia usa la mejor evidencia disponible, y la integra con el buen juicio clínico, y las preferencias del paciente. Procediendo de este modo lograremos dos cosas: implementar las intervenciones correctas para obtener un mejor resultado clínico, y a la vez no efectuar intervenciones que produzcan daño.

En el área de la medicina materno-fetal, cuando tomamos las decisiones respecto a las intervenciones prudentes y necesarias a implementar durante el trabajo de parto, atención del parto y el puerperio inmediato, debemos seguir el camino de la medicina basada en evidencia, conocer los beneficios y riesgos de las intervenciones, para adoptar con las parejas el mejor plan posible. Entre las intervenciones que hemos analizado bajo este prisma, se encuentra el parto planeado para ocurrir en el domicilio.

La atención del parto en la casa, fue la práctica habitual por siglos en la historia de la humanidad; eran tiempos en que las tasas de morbilidad y mortalidad materna y perinatal eran muy altas. La atención médica del parto en el hospital, redujo enormemente los riesgos asociados al parto. En nuestros días, algunas parejas desean volver al parto domiciliario por preferir la intimidad del hogar, evitando una experiencia centrada en la tecnología. En los países y sistemas de salud en donde esta opción está aceptada y regulada, el parto puede ser programado en el domicilio solo en mujeres/embarazos de bajo riesgo.

Los estudios observacionales sugieren que el parto domiciliario reporta menores intervenciones, y no se asocia a alteraciones del resultado perinatal en multíparas; mientras que para primíparas el parto domiciliario resultaría en menores intervenciones, pero a expensas de un peor resultado perinatal (OR 1.75, IC 95\% 1.07-2.86; para mal resultado perinatal en parto domiciliario comparado con hospitalario) 1 .

Solo dos estudios randomizados, controlados, evalúan la seguridad del parto en el domicilio, comparado con el parto en el hospital; sus resultados fueron compilados en una revisión sistemática cuya conclusión es que el bajísimo número de mujeres incluidas (11 mujeres), impide obtener conclusiones confiables 2.

Un reciente estudio de cohorte evaluó los partos ocurridos en Oregon entre 2012 y 2013 , cuyo sistema de registro, permite conocer el resultado de aquellas mujeres que habían planeado su parto en el domicilio o en el hospital. Se observó que el grupo de parto domiciliario vs el de parto hospitalario, presentó un aumento en: la tasa de muerte perinatal $(3,9 / 1.000$ vs 1,8/1.000; OR 2,43; IC 95\% 1,37-4,30), la tasa de convulsiones neonatales $(1,3 / 1.000$ vs $0,4 / 1.000$; OR 3.6; IC 95\% 1.36- 9.50) y la necesidad de transfusiones maternas $(0,6 \%$ vs $0,4 \%$ OR 1.91 ; IC $95 \%$ 1.25-2.93), con una disminución en: tasa de parto vaginal asistido (1\% vs 3\%), tasa de cesárea (5,3\% vs 23,4\%), inducción y aceleración del trabajo de parto 3 .

Estos son los hechos, el parto programado para ocurrir en el domicilio se asocia a mayor riesgo de muerte y enfermedad para el feto o recién nacido y su madre. Estos hechos deben ser conocidos por todas aquellas personas que aspiren a tener el parto en su casa. Tal como nos recomienda Russell debemos 
basar nuestro trabajo en los hechos, y no en lo que queremos que suceda para satisfacer nuestras personales creencias.

El que el parto domiciliario se haya reservado solo para "mujeres/embarazos de bajo riesgo", es una suerte de demostración de que programar el parto en este escenario aumenta el riesgo de tener problemas, y por ello no es admisible para cualquier mujer. Como Sociedad Chilena de Obstetricia y Ginecología y su Rama de Medicina Materno Fetal, no recomendamos la programación de partos en el domicilio, sino que en la seguridad de un recinto hospitalario. En esos sitios, también es posible reducir el número de intervenciones médicas que no sean indispensables, y crear un ambiente confortable para las parejas, que simule la paz y tranquilidad de un hogar, pero con la seguridad necesaria para obtener un recién nacido y madre saludables. La tasa de cesáreas y de inducciones no justificadas médicamente debe disminuir en nuestro país, pero la programación del parto en el domicilio no es el camino correcto para lograrlo.

1. Birthplace in England Collaborative G, Brocklehurst $P$, Hardy $P$, Hollowell $J$, Linsell $L$, Macfarlane A, McCourt C, Marlow N, Miller A, Newburn M, Petrou S, Puddicombe D, Redshaw M, Rowe R, Sandall J, Silverton L \& Stewart M. (2011). Perinatal and maternal outcomes by planned place of birth for healthy women with low risk pregnancies: the Birthplace in England national prospective cohort study. BMJ. 343, d7400.

2. Olsen O \& Clausen JA. (2012). Planned hospital birth versus planned home birth. The Cochrane database of systematic reviews, CD000352.

3. Snowden JM, Tilden EL, Snyder J, Quigley B, Caughey AB \& Cheng YW. (2015). Planned Out-ofHospital Birth and Birth Outcomes. The New England Journal of Medicine. 373, 2642-2653.
Jorge A. Carvajal

Presidente Rama de MMF

SOCHOG Profesor Asociado

Departamento de Obstetricia y Ginecología

Director de Extensión, Educación Continua y Relaciones Internacionales

Escuela de Medicina. Facultad de Medicina Pontificia

Universidad Católica de Chile. 